\title{
Changes in the structure of tethered chain molecules as predicted by density functional approach ${ }^{*}$
}

\author{
M. Borówkd1, A. Patrykiejew11, O. Pizid 2 , S. Sokołowsk ${ }^{11}$ \\ 1 Department for the Modelling of Physico-Chemical Processes, \\ Maria Curie-Skłodowska University, 20031 Lublin, Poland \\ 2 Instituto de Química, Universidad Nacional Autonoma de México, Circuito Exterior, \\ Ciudad Universitaria, 04360, México, D.F., México
}

Received April 1, 2011, in final form June 16, 2011

We use a version of the density functional theory to study the changes in the height of the tethered layer of chains built of jointed spherical segments with the change of the length and surface density of chains. For the model in which the interactions between segments and solvent molecules are the same as between solvent molecules we have discovered two effects that have not been observed in previous studies. Under certain conditions and for low surface concentrations of the chains, the height of the pinned layer may attain a minimum. Moreover, for some systems we observe that when the temperature increases, the height of the layer of chains may decrease.

Key words: brush, adsorption, density functional theory, scaling

PACS: 68.47.Mn, 61.25.H-, 68.47.Pe, 82.35.Gh

\section{Introduction}

The materials modified with tethered chains play an important role in several technological fields. With the proper choice of the grafting surface, the length of chains and the grafting density, one can obtain novel products exhibiting desired behavior. The materials modified in such a way are utilized in a wide range of applications including chromatography, adhesion, lubrication and colloidal stabilization [1 3 ]. Recent studies have focused on the development of "smart" materials, whose properties can be readily altered by applied stimuli. They can find applications in drug delivery, as chemical sensors and in controlling the nanoparticle transport [4 7]. Furthermore, applications in nanotechnology include end grafted polymers on nanopatterned surfaces, on nanoparticles, or on carbon nanotubes [8 12 ].

Much effort has been directed toward theoretical description of grafted polymer layers. The studies included scaling theories [13-15], classical self-consistent field methods [16, 17], single chain mean-field methods [18], density functional theories [18 -22] and computer simulations [23 34]. In our opinion, particularly important for further theoretical studies of systems involving tethered chains is the development of density functional theory (DFT) [20, 21, 35 42] that is based on the approach proposed by $\mathrm{Yu}$ and $\mathrm{Wu}$ [43]. In general, this type of theory represents a powerful tool for the study of adsorption and phase behavior of confined systems. Within this approach, theoretical description of "pinning" the terminating segments of chain molecules to the walls can be realized by using the ideas of the previously developed methods of bonding the fluid species to the walls in an associative treatment [44, 45].

Recent theoretical works based on the DFT [20, 21, 35-42] have mainly concentrated on the description of adsorption of fluids on modified surfaces, while the problem of the changes in the layer of pinned chains itself has attracted less attention. Of course, the accumulation of fluid molecules near the adsorbing surface induces changes in the tethered layer, especially when the

${ }^{*}$ Dedicated to Professor Yu. Kalyuzhnyi on the occasion of his 60th birthday. 
adsorbed fluid undergoes prewetting of layering transitions [40, 42]. However, no systematic density functional study of changes in the structure of tethered chains has been undertaken so far.

The problem of a change of the configuration of tethered chains with their length, surface density and the quality of a solvent has been considered in a great number of publications. One of the first theoretical works devoted to that problem were the publications by Alexander and de Gennes 13 , 14]. However, the references to numerous more recent works can be found in the review article of Descas et al. [46] and in the $\mathrm{PhD}$ thesis by Chremos [47]. We know that in a good solvent, for not too high surface densities of tethered chains and when the free segments (i.e. all but the tethered segment) are not too strongly attracted by the surface, the chains assume the so-called mushroom configuration. With an increase of the surface density, the overlapping mushroom configuration is observed. This configuration precedes the brush regime that occurs at high surface concentrations. Despite the great number of experimental, theoretical and computational investigations on the behavior of end anchored chains, the quantitative determination of the parameter space appropriate for detailed studies of transitions between possible configurations of tethered chains is still vague. In addition, the predictions of theories do not always coincide with the results of simulations [32, 48]. Moreover, in our opinion insufficient attention has been paid to some problems, such as the effect of competition between strong adsorption of fluid molecules and adsorption of free segments of a chain on the structure of the chains and to the effect of the architecture of branched polymers on the structure of the formed layer [49].

The description of systems comprising tethered chains at a microscopic level requires the knowledge of numerous parameters. One has to know not only the surface density and the length of the chains, "quality" (and density) of the solvent and the temperature, but also details about the architecture of the chains, parameters describing the interactions between all the components themselves and between the components and the surface, and, in some cases, the topography of the end anchored chains on the surface. A huge number of parameters of the model delimits the possibility of an effective scanning of the space of the model parameters for a precise evaluation of the regions of different configurations of chains and the transitions between them by long lasting computer simulations. Such studies, however, can be carried out by using theoretical approaches based on microscopic models. Therefore, the aim of the present work is to employ the DFT used in our previously works $[20,21,39-42]$ to investigate how the thickness of the pinned layer changes with their length and with their surface density. Our principal purpose is to check whether the theory employed by us is capable of reproducing the results of coarse-grained approaches [13 15]. We are also looking for some new effects that have not been observed in previous studies.

\section{Theory}

Following our previous works [20, 21, 39, 42] we consider a fluid of spherical molecules of diameter $\sigma_{\mathrm{f}}$ in contact with a modified substrate. The surface is covered by the film of preadsorbed chain molecules. All the chains are represented by tangentially jointed $M$ spherical beads of the same diameter, $\sigma_{\mathrm{c}}$. The chain connectivity is enforced by the bonding potential between nearestneighbor segments, $V_{\mathrm{b}}$. This potential satisfies the equation [43]

$$
\exp \left[-\beta V_{\mathrm{b}}(\mathbf{R})\right]=\prod_{i=1}^{M-1} \delta\left(\left|r_{i}-r_{i+1}\right|-\sigma_{\mathrm{c}}\right) / 4 \pi \sigma_{\mathrm{c}}
$$

where $\mathbf{R}=\left(\mathbf{r}_{1}, \mathbf{r}_{2}, \ldots, \mathbf{r}_{M}\right)$ is a vector specifying segment positions of consecutive segments. Each polymer molecule of the chemically bonded phase contains one surface-binding segment located at its end (indexed as "1") that interacts with the wall via the potential

$$
\exp \left[-\beta v_{\mathrm{s} 1}(z)\right]=C \delta\left(z-\sigma_{\mathrm{c}} / 2\right)
$$

where $z$ is a distance from the surface and $C$ is a constant. This potential implies that the surfacebinding segments always lie at the distance $x=\sigma_{\mathrm{c}} / 2$ from the surface. The remaining segments of 
the grafted molecules $(j=2,3, \ldots, M)$ are "neutral" with respect to the surface, i.e., they interact with the surface via a hard-wall potential

$$
v_{\mathrm{s} j}(z)= \begin{cases}\infty, \quad z<\sigma_{\mathrm{c}} / 2 \\ 0, \quad z \geqslant \sigma_{\mathrm{c}} / 2\end{cases}
$$

The fluid molecules, however, interact with the surface via the Lennard-Jones $(9,3)$ potential of the form.

$$
v(z)=\varepsilon_{\mathrm{fs}}\left[\left(z / z_{0}\right)^{9}-\left(z / z_{0}\right)^{3}\right]
$$

with $z_{0}=\sigma_{\mathrm{f}} / 2$

We assume the Lennard-Jones $(12,6)$ type interactions between all segments, between the segments and fluid particles and between the fluid particles

$$
u_{i j}(r)=\varepsilon_{i j}\left[\left(r / \sigma_{i j}\right)^{12}-\left(r / \sigma_{i j}\right)^{6}\right] \text {, }
$$

where $\sigma_{i j}=0.5\left(\sigma_{i}+\sigma_{j}\right), i, j=c, f$. Employing the perturbative treatment we split these interactions into the repulsive (reference) and attractive (perturbation) parts according to the WeeksChandler-Anderson scheme [50] $u_{i j}(r)=u_{i j, \text { ref }}(r)+u_{i j \text {,pert }}(r)$, where

$$
u_{i j, \text { pert }}(r)= \begin{cases}-\varepsilon_{i j}, & r<2^{1 / 6} \sigma_{i j} \\ u_{i j}(r), & r \geqslant 2^{1 / 5} \sigma_{i j}\end{cases}
$$

In order to proceed, let us introduce the notation, $\rho^{(c)}(\mathbf{R})$ and $\rho(z)$, for the density distribution of chains and of spherical species, respectively. However, the theory is constructed in terms of the density of particular segments of chains, $\rho_{\mathrm{s} j}(z)$, and the total segment density of chains, $\rho_{\mathrm{s}}(z)$. These densities are introduced via commonly used relations, see the original development in [20, 21, 39 42 ]

$$
\rho_{\mathrm{s}}(\mathbf{r})=\sum_{j=1}^{M} \rho_{\mathrm{s} j}(\mathbf{r})=\sum_{j=1}^{M} \int \mathrm{d} \mathbf{R} \delta\left(\mathbf{r}_{j}-\mathbf{r}\right) \rho^{(c)}(\mathbf{R}) .
$$

In the system under study all the local densities are the functions of the distance from the surface only.

The system is studied in a grand canonical ensemble with the constraint of constancy of the number of chain molecules, i.e.

$$
\rho_{\mathrm{c}}=\int \mathrm{d} z \rho_{\mathrm{s} j}(z)
$$

where $\rho_{\mathrm{c}}$ is the number of chain molecules per area of the surface, i.e. it is the surface density of the chains. Of course, the integral in equation (8) does not depend on the segment index $j$.

The thermodynamic potential appropriate to the description of the system is

$$
Y=F\left[\rho_{\mathrm{s}}(z), \rho(z)\right]+\int \mathrm{d} z \rho(z)[v(z)-\mu]+\sum_{j=1}^{M} \int \mathrm{d} z \rho_{\mathrm{s} j} v_{\mathrm{s} j}(z)
$$

where $F\left[\rho_{\mathrm{s}}(z), \rho(z)\right]$ is the Helmholtz free energy functional and $\mu$ is the chemical potential of the reference system fluid. The expression for $F\left[\rho_{\mathrm{S}}(z), \rho(z)\right]$ is obtained from the theory described in our earlier works [20, 21, 39 42] and in the original papers by $\mathrm{Yu}$ and $\mathrm{Wu}$ [43]. The density profile of fluid molecules and the segment density profiles are obtained by minimizing the functional (9) under the constraint (8). For the sake of brevity we do not present the resulting density profile equations. They can be found in the above cited publications. We should only note that the reference system comprises only fluid species. The chemical potential of the fluid, $\mu$ is thus given by

$$
\mu=k T \ln \rho_{\mathrm{b}}+\mu_{\mathrm{hs}}+\rho_{\mathrm{b}} \int u_{\mathrm{ff}, \text { pert }}(r) \mathrm{d} \mathbf{r},
$$


where $\rho_{\mathrm{b}}$ is the reference system density and $\mu_{\mathrm{hs}}$ is the chemical potential of hard-spheres of diameter $\sigma_{\mathrm{f}}$, resulting from the Carnahan-Starling equation of state.

The system under study is described by several parameters. In order to reduce the number of parameters to a minimum, we assume that $\sigma_{\mathrm{f}}=\sigma_{\mathrm{c}}=\sigma_{\mathrm{cf}} \equiv \sigma$ and that $\varepsilon_{\mathrm{ff}}=\varepsilon_{\mathrm{cf}} \equiv \varepsilon$. The Lennard-Jones $(12,6)$ potential parameter, characterizing the interactions between segments of the chain, $\varepsilon_{\mathrm{cc}}$, as well as the fluid-solid energy parameter are expressed in the units of $\varepsilon$, $\varepsilon_{\mathrm{cc}}^{*}=\varepsilon_{\mathrm{cc}} / \varepsilon$ and $\varepsilon_{\mathrm{fs}}^{*}=\varepsilon_{\mathrm{fs}} / \varepsilon$. The reduced densities are defined as usual, $\rho_{\mathrm{b}}^{*}=\rho_{\mathrm{b}} \sigma^{3}, \rho^{*}(z)=\rho(z) \sigma^{3}$, $\rho_{\mathrm{s} j}^{*}(z)=\rho_{\mathrm{s} j}(z) \sigma^{3}, \rho_{\mathrm{s}}^{*}(z)=\rho_{\mathrm{s}}(z) \sigma^{3}$ and $\rho_{\mathrm{c}}^{*}=\rho_{\mathrm{c}} \sigma^{2}$. Moreover, we introduce the reduced temperature as $T^{*}=k T / \varepsilon$.

Almost all the calculations (unless otherwise explicitly stated) have been carried out for the fluid bulk density $\rho_{\mathrm{b}}^{*}=0.66$. This density nearly coincides with the liquid density on the liquid-vapor coexistence curve at $T^{*}=1$.

\section{Results and discussion}

Before discussing the results we should emphasize that it is unclear how to determine the brush height in a precise meaning from the density profiles obtained via DFT or from simulations. It has been a widespread practice to define the brush height, $\langle h\rangle$, from the first moment of the total segment density profile [26, 51, 52]

$$
\langle h\rangle=a \frac{\int \mathrm{d} z z \rho_{\mathrm{s}}(z)}{\int \mathrm{d} z \rho_{\mathrm{s}}(z)} .
$$

Usually, (cf. [26, 51]) the coefficient $a$ was set to be $a=8 / 3$, but also $a=2$ was used (see, e.g. [52]) and the latter value has been adopted in our calculations. Moreover, usually the height of the brush, as well as the surface density of tethered chains were scaled by the radius of gyration (or by some powers of the radius of gyration) of the chain molecules, cf. [26, 32, 48, 51, 52]. Unfortunately, the value of the radius of gyration cannot be self-consistently obtained within the framework of the considered approach. Therefore, in our calculations we use the reduced units as defined at the end of the previous section.

\subsection{Change of the brush height with the length of chains}

We begin with the discussion of the changes of the brush height with the length of the tethered chains. In figure 1 we show the plots of $\langle h\rangle / \sigma$ versus $M$ for a number of systems characterized by different values of the intermolecular potentials and obtained for different surface grafting densities. In part (a) the solid lines were obtained assuming weak interactions between fluid molecules and solid surface, $\varepsilon_{\mathrm{fs}}^{*}=\varepsilon_{\mathrm{fs}} / \varepsilon=1$. The remaining lines were evaluated for solid surfaces attracting the fluid molecules more strongly, see the figure caption. The surface density of grafted chains is $\rho_{\mathrm{c}}^{*}=0.1$ for all, but the one curve, for which $\rho_{\mathrm{c}}^{*}=0.02$ (the solid line decorated with solid circles). From first glance, for $M>10$, almost all the curves plotted here are well approximated by straight lines (an exception is the solid line decorated with solid circles, calculated for $\rho_{\mathrm{c}}^{*}=0.02$ ). However, much better approximations are obtained having assumed a power-law dependence, cf. [53]

$$
\langle h\rangle \propto M^{\alpha} .
$$

Even for the segment-segment interactions of a hard-sphere type (solid line decorated with stars) the approximation of numerical results by a straight line of the form $\langle h\rangle=a+b M$ yields the correlation coefficient $R_{\mathrm{c}}$ of the order of $R_{\mathrm{c}} \approx 0.990$, while the use of equation (12) gives $R_{\mathrm{c}} \approx 0.9999$ for $\alpha=0.9134$.

When the attractive interactions between the segments are turned on, the exponent $\alpha$ decreases. For $\varepsilon_{\mathrm{cc}}=0.6 \varepsilon$ we obtain $\alpha=0.850$ (solid line with crosses; the correlation coefficient in this case is again of the order of 0.9999), while for $\varepsilon_{\mathrm{cc}}=\varepsilon$ we have $\alpha=0.738$ (solid line with open squares; the correlation coefficient is now slightly lower, $\left.R_{\mathrm{c}} \approx 0.999\right)$. An increase of the fluid-solid energy decreases slightly the value of $\alpha$. For example, when $\varepsilon_{\mathrm{cc}}=0.6 \varepsilon$ and $\varepsilon_{\mathrm{fs}}^{*}=12$ (dashed line with 


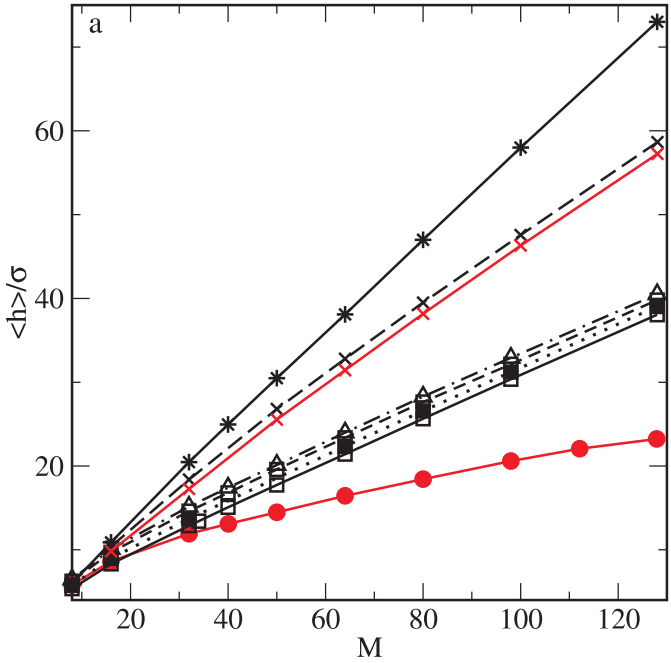

(a)

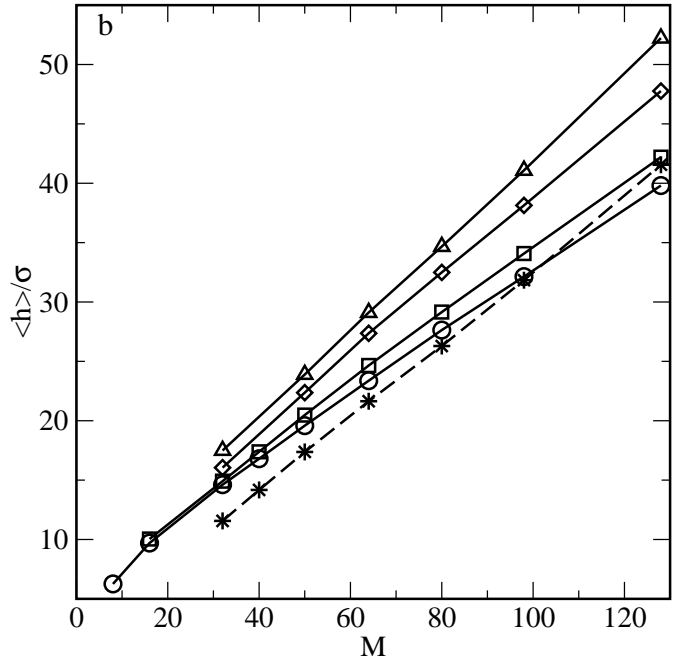

(b)

Figure 1. The dependence of $\langle h\rangle / \sigma$ on $M$. In part (a) the nomenclature of the lines decorated with different symbols is as follows. Solid lines have been evaluated for $\varepsilon_{\mathrm{fs}}^{*}=1$ and for the chains built of hard-spheres, $\varepsilon_{\mathrm{cc}}^{*}=0$ (stars); $\varepsilon_{\mathrm{cc}}^{*}=0.6$ (crosses) $\varepsilon_{\mathrm{cc}}^{*}=1$ (open squares). Dotted line with filled squares is for $\varepsilon_{\mathrm{fs}}^{*}=5$ and $\varepsilon_{\mathrm{cc}}^{*}=1$. Dashed lines are for $\varepsilon_{\mathrm{fs}}^{*}=12$ and for $\varepsilon_{\mathrm{cc}}^{*}=0.6$ (crosses) and $\varepsilon_{\mathrm{cc}}^{*}=1$ (open squares), while dash-dotted line with triangles is for $\varepsilon_{\mathrm{fs}}^{*}=18$ and $\varepsilon_{\mathrm{cc}}^{*}=1$. The values of the remaining parameters are: $T^{*}=1$ and $\rho_{\mathrm{c}}^{*}=0.1$. Additionally, solid line with filled circles has been obtained for $\varepsilon_{\mathrm{fs}}^{*}=1$ and $\varepsilon_{\mathrm{cc}}^{*}=1, T^{*}=1$ and $\rho_{\mathrm{c}}^{*}=0.02$. In part (b) we show the effect of surface density $\rho_{\mathrm{c}}^{*}$ and of the temperature on the evaluated curves $\langle h\rangle / \sigma=f(M)$. Solid lines are at $T^{*}=1$ and $\rho_{\mathrm{c}}^{*}=0.1$ (open circles), $\rho_{\mathrm{c}}^{*}=0.12$ (open squares), $\rho_{\mathrm{c}}^{*}=0.15$ (open diamonds) and $\rho_{\mathrm{c}}^{*}=0.20$ (open triangles). The dashed line with stars is for $\rho_{\mathrm{c}}^{*}=0.1$ and at $T^{*}=4$. The values of the remaining parameters are $\varepsilon_{\mathrm{fs}}^{*}=12$ and $\varepsilon_{\mathrm{cc}}^{*}=1$.

crosses) we have $\alpha=0.834$, instead of $\alpha=0.850$ for $\varepsilon_{\mathrm{fs}}^{*}=1$ (solid line with crosses). Similarly, for $\varepsilon_{\mathrm{cc}}=\varepsilon$ and for $\varepsilon_{\mathrm{fs}}^{*}=18$ (dash-dotted line with triangles) we obtain $\alpha=0.720$. The last value is lower than that obtained for $\varepsilon_{\mathrm{cc}}=\varepsilon$ and $\varepsilon_{\mathrm{fs}}^{*}=1$. The effect of the parameter $\varepsilon_{\mathrm{fs}}^{*}$ on the exponent $\alpha$ is much weaker that of the parameter $\varepsilon_{\mathrm{cc}}$.

The solid line decorated with filled circles that has been evaluated for much lower surface density of tethered chains deviates very much from all the remaining results. In this case the exponent of equation $(12)$ is much lower and equals $\alpha \approx 0.49$. This indicates that the surface density of tethered chains greatly effects the scaling relationship (12). To investigate this problem, we calculated the values of $\langle h\rangle / \sigma$ for different values of $\rho_{\mathrm{c}}^{*}$, keeping the values of the following parameters constant: $\varepsilon_{\mathrm{cc}}=\varepsilon$ and $\varepsilon_{\mathrm{fs}}^{*}=12$, see figure 1 (b). Solid lines displayed here correspond to $T^{*}=1$, while the dashed line is for $T^{*}=4$. For $\rho_{\mathrm{c}}^{*}=0.1$ (solid line with open circles) we found that $\alpha=0.729$, while for $\rho_{\mathrm{c}}^{*}=0.2$ (solid linear with triangles) $-\alpha=0.828$. Therefore, we conclude that an increase of the surface density of tethered chains increases the value of $\alpha$. This is a rather obvious result because in the case of higher values of $\rho_{\mathrm{c}}^{*}$ the chains are more stretched and there is "less room" for lateral motions of the segments which could possibly lead to "coiling" the chains. However, the effect of the temperature on the exponent $\alpha$ is not so obvious. The dashed line was obtained at $T^{*}=4$ for $\rho_{\mathrm{c}}^{*}=0.1$. Now, $\alpha \approx 0.90$. For lower values of $M$ the height of the brush at $T^{*}=4$ is lower than at $T^{*}=1$, but for longer chains the situation is reverse. One can argue here that the average brush height, $\langle h\rangle$, should be related to the end-to-end distance, $R_{1-N}$, of a single polymer chain, at least at low grafting density. Unfortunately, the density functional approach used in this work does not allow for the evaluation of the end-to-end distance, and therefore we cannot carry out any test for a relation between $\langle h\rangle$ and $R_{1-N}$ using self-consistent data in the framework of the applied theory. 
The configuration assumed by the chains depends on entropic as well as on energetic contributions to the free energy. The chains maximize their configurational entropy by adopting a randomwalk-like configurations. However, depending on the energies of segment-segment and segment-fluid attractions, they tend to maximize the contact between relevant species. Moreover, the attraction between fluid molecules and the solid leads to accumulation of the fluid molecules in the vicinity of the solid surface. At $T^{*}=4$ the Boltzmann factor of the fluid-solid interactions is much lower than at $T^{*}=1$. Therefore, the accumulation of fluid molecules and thus the effect of "superseding" the segments from the region close to the wall is smaller at $T^{*}=4$. The adsorption of fluid molecules on the brush structure plays a more significant role for shorter chains. For longer chains, however, the entropic effects prevail and at $T^{*}=4$ the brush becomes more stretched than at $T^{*}=1$. We return to the problem of temperature dependence of the brush height hereinbelow.

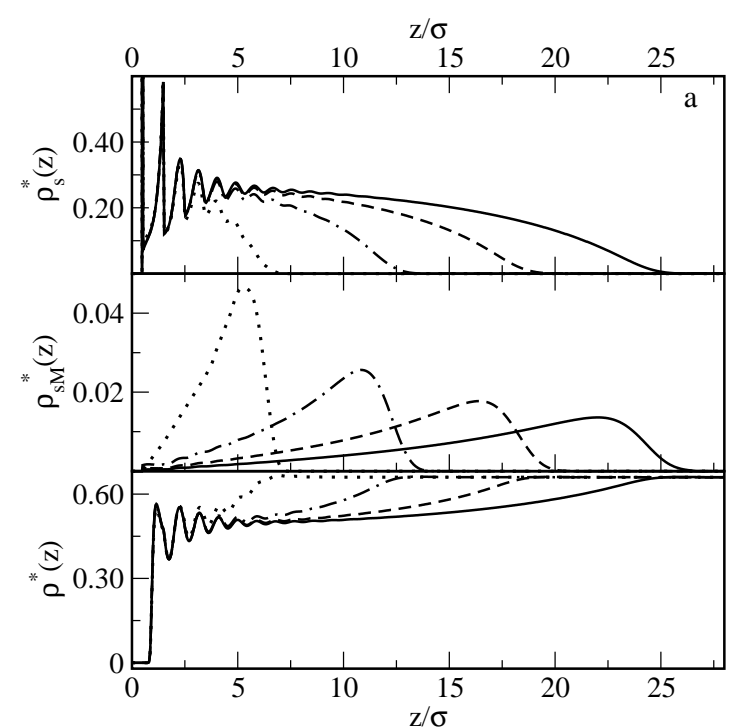

(a)

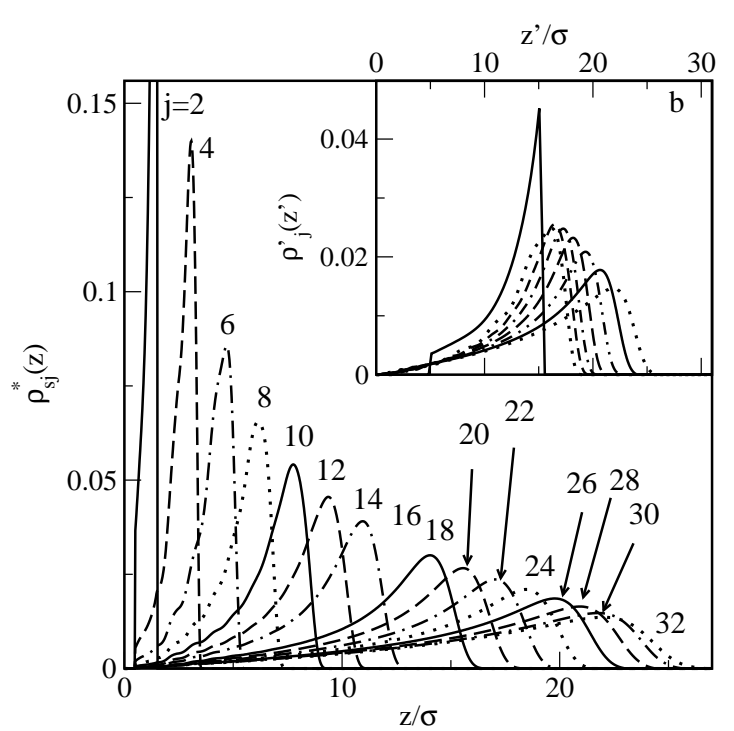

(b)

Figure 2. Part (a). Total segment density profiles (upper panel), the density profiles of the last segment (middle panel) and the fluid density profiles for the chains of different length. Dotted line is for $M=8$, dash-dotted line - for $M=16$, dashed line is for $M=24$ and solid line is for $M=32$. Part (b) shows the density profiles of individual segments, $\rho_{\mathrm{s} j}^{*}(z)$ for the values of $j$ given in the figure. Here the chain length is $M=32$. The inset to part (b) shows the scaled segment density profiles, as described in the text. The consecutive curves from left to right are for $j=2,6,10,14,18,22,26$ and 30 . The segment-segment interactions are of hard-sphere type, $\rho_{\mathrm{c}}^{*}=0.15, T^{*}=1$ and $\varepsilon_{\mathrm{fs}}^{*}=1$.

We have monitored the changes in the structures of the brush and of the fluid with the changes of the parameters of the model by inspecting the density profiles. Examples of the obtained results are shown in figures 2 and 3 . Figure 2 (a) shows how the total density profiles (the upper panel), the profiles of the last segment (the middle panel) and the fluid density profiles (the lower panel) change with the length of tethered chains. We see that if the length of the chains increases, the initial parts (i.e. the part close to the wall) of the total segment density profiles and of the fluid density profiles remain almost the same as for shortened chains. An increase in the number of segments $M$ causes expansion of the region where the segments and the fluid molecules exhibit a layered structure. Instantaneously, the "tails" of the profiles become more diffuse for longer chains. In the middle part of figure 2 (a) we also see that even for long chains there is nonzero probability of finding the last segments in the vicinity of the wall. Such a shape of the segment density profiles suggest coiling of the chains which can even lead to the formation of loops with both terminating segments of a chain located at the wall. 
Figure 2 (b) displays the profiles of individual segments with even numbers for tethered 32mers. With an increase of the segment number, the profiles become more and more diffuse. According to classical self-consistent field theory, the re-scaled distributions of the segments $\rho_{j}^{\prime}\left(z^{\prime}\right)=$ $\rho_{\mathrm{s} j}^{*}(z) \sin [\pi j / 2 M]$ should be an universal function of $z^{\prime}=z / \sin [\pi j / 2 M]$, i.e., the plot of $\rho_{j}^{\prime}$ versus $z^{\prime}$ should be independent of the segment index $j \quad$ [54 56]. The inset to figure 2 (b) provides a test whether the above scaling can be also applied to the DFT results. Evidently, the self-consistent field scaling fails when applied to the density functional results of this work.

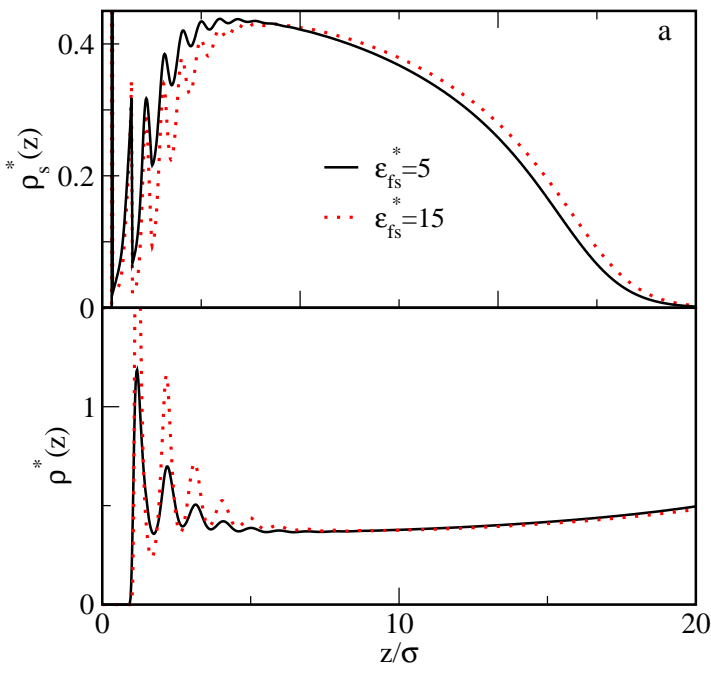

(a)

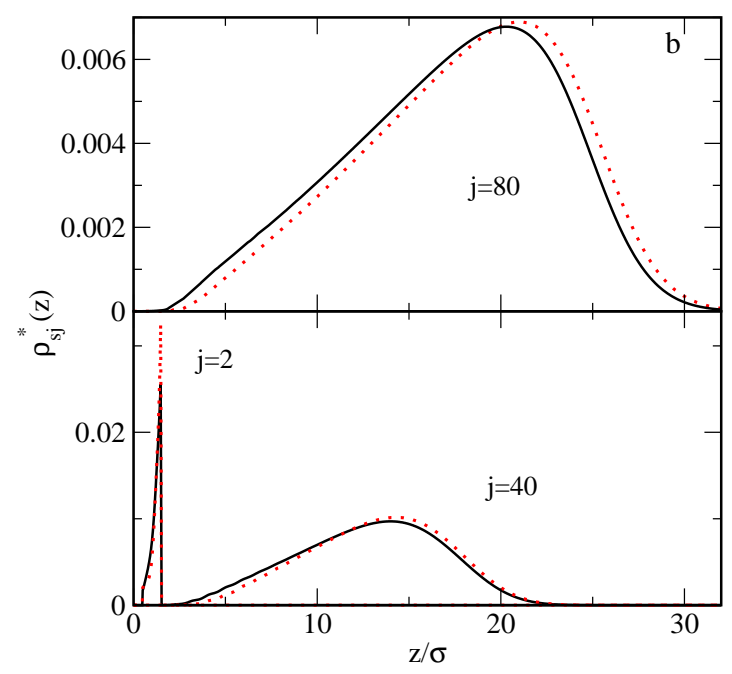

(b)

Figure 3. Part (a). Total segment density profiles (upper panel) and fluid density profiles (lower panel) of $M=80$-mers for $\varepsilon_{\mathrm{fs}}^{*}=5$ (solid lines) and $\varepsilon_{\mathrm{fs}}^{*}=15$ (dotted lines). In part (b) we display the profiles of the last segment (upper panel) and of the middle segment $(j=40)$ and of the second segment, $j=2$ (the latter are divided by 10). The values of the remaining parameters are $R_{\mathrm{c}}^{*}=0.1, T^{*}=1$ and $\varepsilon_{\mathrm{cc}}=\varepsilon$.

Figure 3 (a) illustrates the change of the total segment density profile of tethered chains and of the profile of the fluid (lower and upper panels, respectively), while figure 3 (b) shows the changes of the last (lower panel) and of the middle (upper panel) with an increase of the fluid-solid attraction. We see that when $\varepsilon_{\mathrm{fs}}^{*}$ increases, the total segment density profile is pushed away from the solid surface by the adsorbing fluid. This effect is more pronounced for the last segment than for the initial one of middle segments of the chain (figure 3 (b)).

\subsection{The role of the surface density of the chains}

The next problem we want to study in detail is how the surface density of the pinned chains effects the height of tethered layer. We begin our discussion with the results for rather short chains built of 16-mers (the solids modified with the chains of a comparable length are widely used as column fillings in chromatography). In figure 4 we demonstrate the effect of some selected parameters of the model on the relationship of $\langle h\rangle / \sigma$ upon $\rho_{\mathrm{c}}^{*}$. Three curves displayed in part (a) have been calculated assuming that the segment-segment interactions are the same as the segment fluid and fluid-fluid interactions, $\varepsilon_{\mathrm{cc}}=\varepsilon$. The temperature was kept constant and equal to $T^{*}=1$. The curve decorated with open squares is for the solid that weakly attracts fluid molecules, $\varepsilon_{\mathrm{fs}}^{*}=1$, while the curve decorated with open diamonds is for strongly attracting solid surface, $\varepsilon_{\mathrm{fs}}^{*}=15$. These two curves were evaluated assuming that the fluid density was $\rho_{\mathrm{b}}^{*}=0.66$. However, we also present here the results for tethered chains in a vacuum (curve decorated with solid circles). Part (b) illustrates the effects of weakening of the segment segment interactions and of the temperature on $\langle h\rangle / \sigma$.

For very low surface densities of tethered chains, the brush height remains almost constant. This is not surprising, because under such conditions a tethered chain does not almost "feel" the 


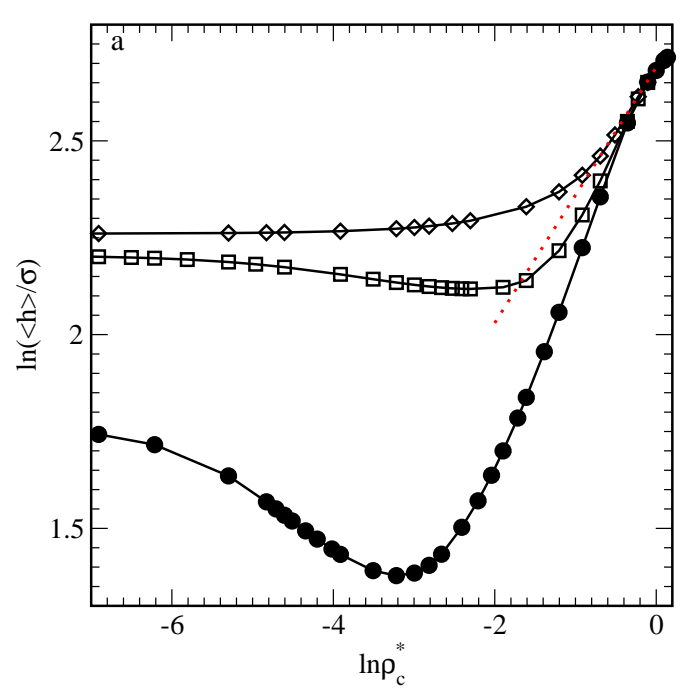

(a)

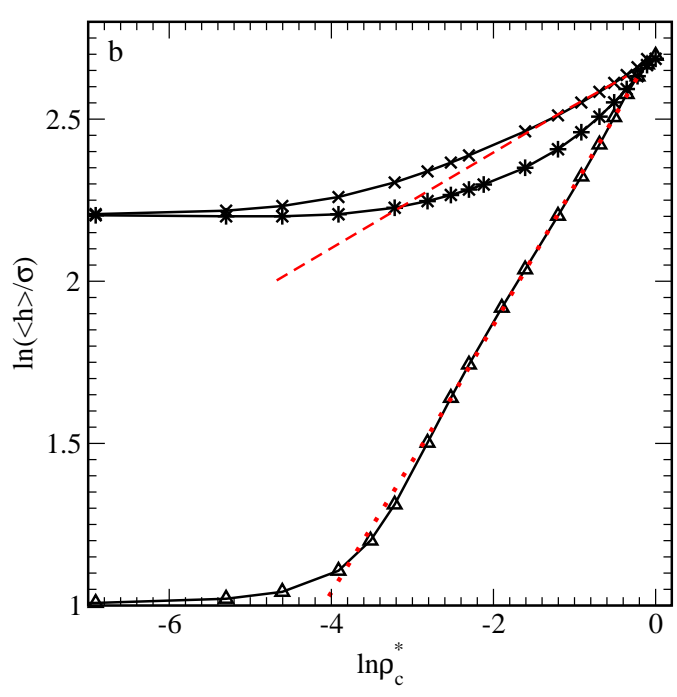

(b)

Figure 4. The dependence of $\langle h\rangle / \sigma$ on the surface density of tethered 16-mers. The nomenclature of the lines in part (a) is as follows: $\varepsilon_{\mathrm{fs}}^{*}=15$ and $\rho_{\mathrm{b}}^{*}=0.66$ - line with open diamonds; $\varepsilon_{\mathrm{fs}}^{*}=1$ and $\rho_{\mathrm{b}}^{*}=0.66-$ line with open squares. The line with solid circles was obtained for the brush in a vacuum, $\rho_{\mathrm{b}}^{*}=0$. In all cases $\varepsilon_{\mathrm{cc}}=\varepsilon$ and $T^{*}=1$. Dotted straight line has the slope of $1 / 3$ and fits all the results in the limit of high $\rho_{\mathrm{c}}^{*}, \rho_{\mathrm{c}}^{*} \in[0.5,0.9]$. In part (b) the consecutive lines were obtained for the following parameters: $\varepsilon_{\mathrm{cc}}=0.6 \varepsilon$ and $T^{*}=1-$ line with stars; $\varepsilon_{\mathrm{cc}}=0$ (hard-sphere segment-segment interactions) and $T^{*}=1$ - line decorated with crosses; $\varepsilon_{\mathrm{cc}}=\varepsilon$ and $T^{*}=5$ - line decorated with triangles. In all cases $\rho_{\mathrm{b}}^{*}=0.66$ and $\varepsilon_{\mathrm{fs}}^{*}=1$. The dotted and dashed straight lines denote the approximations of the log-log plots, as described in the text.

presence of other chains and assumes the configuration that is only effected by the presence of solvent molecules. The accumulation of solvent molecules in the vicinity of the solid surface leads to an increase of $\langle h\rangle / \sigma$. Of course, the last statement is true if the solvent-fluid attraction is similar (in our case identical) to the fluid-fluid attraction. The behavior of the brush in "bad" solvent can be different [34], but this problem requires additional studies.

With further increase of $\rho_{\mathrm{c}}^{*}$ and when the attractive fluid-solid potential is weak, there develops a minimum on the curve of $\langle h\rangle / \sigma$ vs. $\rho_{\mathrm{c}}^{*}$ (see the line with open squares in figure 4 (a)). In this case, the minimum is located at $\rho_{\mathrm{c}}^{*} \approx 0.1$. Similarly, a minimum on the curve of $\langle h\rangle / \sigma$ vs. $\rho_{\mathrm{c}}^{*}$ is observed if the brush is in contact with a vacuum, but now it is located at much lower surface brush density, $\rho_{\mathrm{c}}^{*} \approx 0.04$. For a strongly attractive wall, this minimum disappears (solid line with crosses in figure 4 (a)). Also, the minimum vanishes when the segment-segment attraction is lowered or when the temperature is raised, see figure 4 (b). Evidently, this phenomenon is caused by the segment-segment attractive interactions. At low temperatures, the attraction between segments causes coiling of the chains near the wall. However, in the case of the wall strongly attracting fluid molecules, the coiling is inhibited by the fluid molecules accumulated at the wall.

In general, two characteristic regions on the curves $\langle h\rangle / \sigma$ vs. $\rho_{\mathrm{c}}^{*}$ in figures 4 (a) and 4 (b) can be distinguished: the region of almost constant values of $\langle h\rangle / \sigma$ at low surface densities of tethered chains and the region where the logarithm of the height of the brush changes with $\ln \rho_{\mathrm{c}}^{*}$ almost linearly. Such a behavior was also observed in computer simulations [26, 48].

Classical scaling theories predict that the height of the brush scales with the surface brush density as

$$
\langle h\rangle \propto\left(\rho_{\mathrm{c}}\right)^{\gamma}
$$

with $\gamma=1 / 3$, as predicted by the self-consistent mean-filed theory (see, e.g., [26]), or with $\gamma \approx 0.35$, as predicted by the Alexander-de Gennes approach (see, e.g., [48]. The dotted line plotted in figure 4 (a) has the slope of $1 / 3$ and it well fits all the data for $0.5<\rho_{\mathrm{c}}^{*}<0.9$. However, for the 
values of $\rho_{\mathrm{c}}^{*}$ higher than 0.9 the changes of $\langle h\rangle / \sigma$ are much smaller than predicted by equation (13). Of course, for a very dense brush, its height attains a maximum corresponding to fully stretched chains.

At high temperature, the linearity range of the log-log plot is much wider, cf. dotted line in figure 4 (b). This line well approximates the $\left\langle h\left(\rho_{\mathrm{c}}^{*}\right) / \sigma\right\rangle$ curve evaluated at $T^{*}=5$ (the line with triangles), but now the exponent $\gamma \approx 0.43$ is significantly higher than predicted by the theory of Alexander and de Gennes [13 15]. Similarly, when the segment-segment interactions are of hardsphere type, there exist a range of surface densities $\rho_{\mathrm{c}}^{*}$, within which the log-log plot is linear (dashed line in figure 4 (b)), but now the slope is much lower, $\gamma \approx 0.14$, than predicted by the classical theories. Only when $\varepsilon_{\mathrm{cc}}=0.6 \varepsilon$ (the curve with stars in figure 4 (b)), the straight line approximating the data in a relatively wide range of $\rho_{\mathrm{c}}^{*}$ values has the slope close to $1 / 3$ (the relevant line has been omitted in figure 4 (b)).

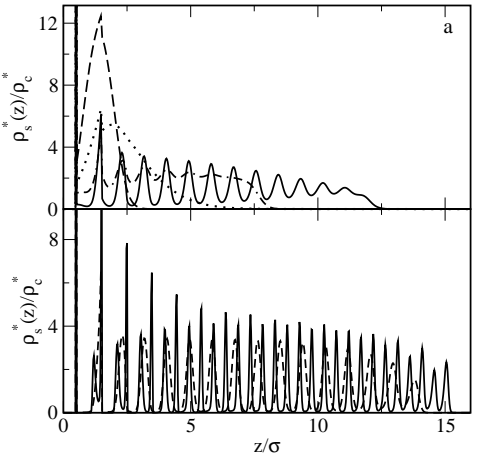

(a)

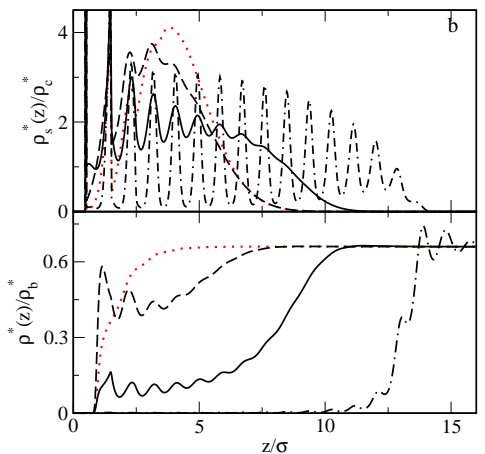

(b)

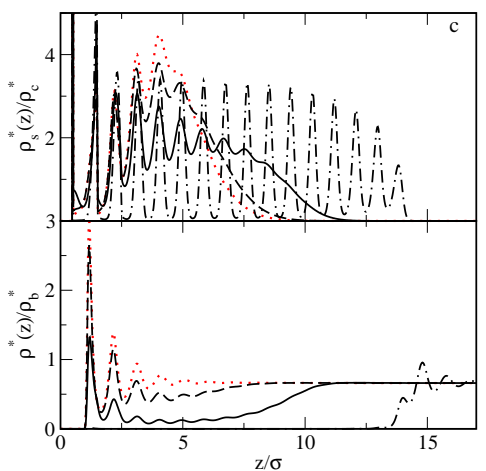

(c)

Figure 5. Examples of the total segment density profiles of 16-mers and the profiles of fluid calculated along the curves displayed in figure 4 (a). Part (a) is for the brush in contact with a vacuum (line with solid circles in figure 4). Upper panel shows the results for $\rho_{\mathrm{c}}^{*}=2 \cdot 10^{-6}$ dotted line, 0.04 (this surface density corresponds to the minimum of $\langle h\rangle / \sigma)$ - dashed line, 0.4 - dash-dotted linear and 0.7 - solid line. Lower panel is for $\rho_{\mathrm{c}}^{*}=0.9$ - dashed line and for 1.15 - solid line. Part (b) was calculated along the line with open squares in figure 4 (a). Upper panel displays the total segment density profiles and lower panel displays the profiles of fluid. The calculations are for $\rho_{\mathrm{c}}^{*}=2 \cdot 10^{-6}$ - dotted lines, 0.1 (this surface density corresponds to the minimum of $\langle h\rangle / \sigma)$ - dashed line, 0.4 - solid line and 0.9 - dash-dotted line. Part (c) was calculated along the line with open squares in figure 4 (a). The abbreviation of the lines and the surface densities of tethered chains are the same as in part (b).

Figure 5 illustrates how the structure of the brush built of 16 -mers in a vacuum changes with $\rho_{c}^{*}$. At extremely low surface density, $\rho_{\mathrm{c}}^{*}=10^{-6}$, the brush extends to the distance around $z / \sigma \approx 5.5$ and the profiles $\rho_{\mathrm{S}}^{*}(z) / \rho_{\mathrm{c}}^{*}$ remain almost unchanged up to $\rho_{\mathrm{c}}^{*} \approx 5 \cdot 10^{-5}$ (the relevant results have been omitted for the sake of brevity). Then, the coiling process starts due to attraction between the segments and the minimum height of the brush is attained at $\rho_{\mathrm{c}}^{*} \approx 0.04$. The profile corresponding to the brush of minimal height is shown by the dashed line in the upper panel of figure [5] (a). This curve demonstrates that the formed structure essentially comprises two layers at the wall. Further increase of $\rho_{c}^{*}$ leads to the brush expansion. Initially, the layer at the wall resembles a liquid-like film, but then a layered structure develops. For $\rho_{\mathrm{c}}^{*}<0.9$, the number of layers is lower than the number of the segments $M$, but at very high values of $\rho_{\mathrm{c}}^{*}$, the number of layers increases up to $2 M$ (see the lower panel in figure 5 (a)). The brush layer tends to assume the solid-like configuration [57]. Unfortunately, one-dimensional density functional theory used in this work fails in that region of the surface brush densities.

The presence of the fluid changes the structure of the brush, cf. figures 5 (b) and 5 (c). When $\rho_{\mathrm{c}}^{*}$ is not too high (say, $\rho_{\mathrm{c}}^{*}<0.4$, the fluid itself forms layered structure, especially for higher values of $\varepsilon_{\mathrm{fs}}^{*}$. The presence of a well-pronounced fluid layers in the vicinity of the wall inhibits coiling of the brush and breaks the development of a minimum on $\left\langle h\left(\rho_{\mathrm{c}}^{*}\right) / \sigma\right\rangle$ curve. The layers of the fluid 
also cause an increase of the brush height, in comparison with the brush in a vacuum. However, for higher surface densities of tethered chains, the fluid molecules are no longer capable of penetrating the interior of the brush. Consequently, for $\rho_{\mathrm{c}}^{*}>0.5$, the heights of brushes formed in the systems with and without the presence of the fluid, become similar and when $\rho_{\mathrm{c}}^{*}$ still increases the values of $\left\langle h\left(\rho_{c}^{*}\right)\right\rangle / \sigma$ nearly flow together. The last statement is true if the segment-segment, segment-fluid and fluid-fluid interactions in the systems characterized by different values of $\rho_{\mathrm{b}}^{*}$ and $\varepsilon_{\mathrm{fs}}^{*}$ are identical (or similar). However, when those interactions are different, then for high values of $\left\langle h\left(\rho_{\mathrm{c}}^{*}\right) / \sigma\right\rangle$, the differences in the brush height in different systems remain significant, cf. figure 4 (b).

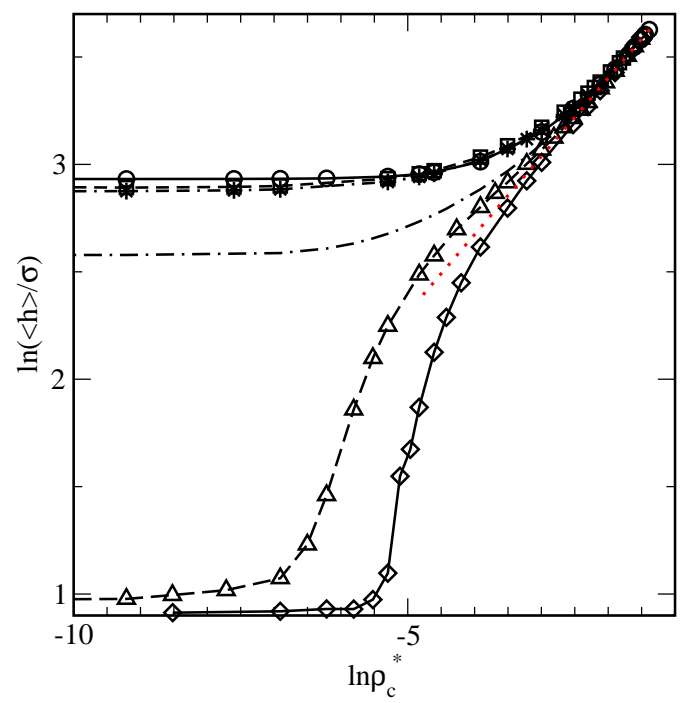

Figure 6. The dependence of $\langle h\rangle / \sigma$ on the surface density of tethered 80-mers. The nomenclature of the lines is as follows: $T^{*}=1-$ solid line with open circles, $T^{*}=2$ - dashed line with open squares, $T^{*}=2.2$ - double dash-dotted line with stars, $T=2.75$ - dash-dotted line, $T^{*}=3-$ dashed line with triangles and $T^{*}=4-$ solid line with diamonds. The calculations are for $\varepsilon_{\mathrm{fs}}^{*}=12$, and $\varepsilon_{\mathrm{cc}}=\varepsilon$. Dotted straight line has the slope 0.35 .

The results presented in figures 4 and 5 have been computed for rather short chains composed of 16 -mers. We now consider the case of 80 -mers. Figure 6 shows the functions $\left\langle h\left(\rho_{\mathrm{c}}^{*}\right) / \sigma\right\rangle$ evaluated at different temperatures for the system characterized by $\varepsilon_{\mathrm{cc}}=\varepsilon$ and $\varepsilon_{\mathrm{fs}}^{*}=12$. Similarly to the case of $M=16$ at low surface densities of grafted chains, we observe here that the heights of the brushes remain almost constant. For high values of $\rho_{c}^{*}$, the curves at different temperatures almost coincide and all of them can be approximated by a straight line with the slope of 0.35 , in accordance with the theory by Alexander and de Gennes. However, we observe here an unexpected effect. Namely, with an increase of the temperature, the average height of the low surface density brush decreases. This decrease is small when the temperature rises from $T^{*}=1$ up to $T=2.2$, but then the changes become larger, especially within the temperature range of $[2.75,3]$. We carefully inspected that region of temperatures and found that the occurring changes are fast, but continuous, i.e., the plot of the thermodynamic potential $Y$ vs $T^{*}$ at a constant $\rho_{\mathrm{c}}^{*}$ does not exhibit any discontinuities.

In order to explain the observed temperature behavior we have inspected the density profiles. In figure 7 we display the segment density profiles of the segment $j=2,40$ and 80 and the total segment density profiles. The calculations were carried out for the system presented in figure 6 at $\rho_{\mathrm{c}}^{*}=0.0001$ and at $T^{*}=2.2$ (solid lines) and at $T^{*}=3$ (dashed lines). Additionally we have displayed here the results evaluated at the same surface density of the chains and at $T^{*}=3$, but assuming that the fluid-solid energy is higher, $\varepsilon_{\mathrm{fs}}^{*}=18$ (dotted lines). The temperature $T^{*}=3$ is well below the bulk critical temperatures of the fluid comprising 80-mers, cf. [58] and thus the chains exhibit a tendency to condensate, even within a single chain. At lower temperature $T^{*}=2.2$ the adsorption of the fluid at the wall is stronger than at $T^{*}=3$. The accumulation of the fluid 


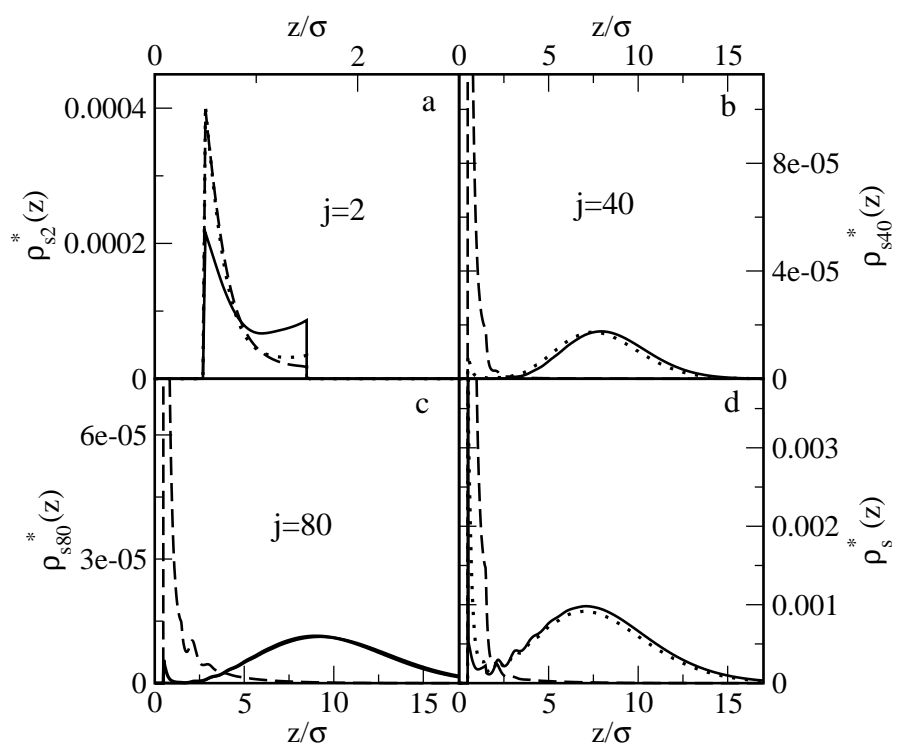

Figure 7. Segment density profiles $\rho_{\mathrm{s} j}(z)$ for $j=2$ (a), $j=40$ (b), $j=80$ (c) and the total segment density profiles (d). The calculations were carried out for the systems from figure 6 at $\rho_{\mathrm{c}}^{*}=0.0001$ and at $T^{*}=2.2$ (solid lines) and at $T^{*}=3$ (dashed lines). Dotted lines show the results evaluated at the same surface density of the chains and at $T^{*}=3$, assuming that $\mathrm{x}$ $\varepsilon_{\mathrm{fs}}^{*}=18$.

particles at the wall causes the situation wherein not all the segments of the chain "condensate", i.e., the initial segments do not join the "cloud" formed by the inner and outer segments and the tethered chains assume a characteristic mushroom-like shape. Indeed, the profiles for $j=40$ and for $j=80$ displayed in figure 7 lie very close to each other and the total segment density profile exhibits a depletion for $0.5<z<2.5$ (this depletion corresponds to the mushroom's "pedicle"). When the temperature is raised up to $T^{*}=3$, a part of the fluid molecules is removed from the surface region, making more room for the segments of the chains. Consequently, the chains are capable of assuming the configuration almost parallel to the wall and then they "condensate" into a thin (in comparison with the mushroom configuration) film covering the wall (pancake configuration) . However, when the wall attracts the fluid particles more strongly, $\varepsilon_{\mathrm{fs}}^{*}=18$, the formation of a thin film of chains at the wall is inhibited by the presence of the fluid particles and the brush assumes a mushroom structure, even at $T^{*}=3$. The phenomenon of the change of the brush structure is connected with an interplay between adsorption of the fluid (the formation of layered structure by the fluid molecules) and the tendency of chains to condensate. In the case of systems under study, this change is a continuous process, but we cannot exclude a situation when this transformation will occur as a first-order transition.

The relations describing the changes in the brush height with the changes of the number of segments and with the grafting density, known as scaling relations $[13-17,26,32,48,52$, were developed using theoretical description based on coarse-grained models. Therefore, it is not surprising that these relations agree well with the coarse-grained simulations (e.g., Monte Carlo simulations that are based on the self-avoiding random walk model of chains), However, when microscopic modelling is applied in computer simulations, several deviations between computer simulation data and scaling theory arise, see, for example [26].

In our calculations we observe that when the strength of the segment-segment interactions decreases, the scaling relations are approximately satisfied within the region of stretched chains. However, more importantly, we have found two unexpected effects. The first one is a possibility of the existence of a minimum on the curve describing the brush height versus the grafting density, while the second one is a decrease of the brush height with an increase of temperature (or an increase 
of the brush height with a decrease of temperature). Both effects have been observed at rather low grafting densities. The second effect results, in our opinion, from temperature dependence of adsorption of solvent molecules. In general, the effect of the adsorption of solvent on the surface on the brush height has been omitted in previous studies. To our best knowledge, there are no relevant computer simulation data that would confirm the existence of the two unusual effects mentioned above. Such simulations require long-lasting calculations and the question whether our observations are artifacts of the theory or they do really exist is currently under study in our laboratory.

\section{Acknowledgements}

S.S. was supported by the Ministry of Science of Poland under Grant No. N N204 151237. M.B., A.P. and O.P. acknowledge support from EC under Grant No. PIRSES 268498.

\section{References}

1. Advincula R.C., Brittain W.J., Caster K.C., Ruhe J., Eds., Polymer Brushes. Wiley VCH, Weinheim, 2004; doi $10.1002 / 3527603824$.

2. Cunliffe D., de las Heras Alarcn C., Peters V., Alexander C., Langmuir, 2003, 19, 288; doi $10.1021 /$ la0263581.

3. Galaev I.Y., Mattiasson B., J. Chromatogr. A, 1994, 662, 27; doi 10.1016/0021-9673(94)85292-8.

4. Minko S., Patil S., Datsyuk V., Simon F., Eichhorn K.-J., Motornov M., Usov D., Tokarev I., Stamm M., Langmuir, 2002, 18, 289; doi $10.1021 /$ la015637q.

5. Russell T.P., Science, 2002, 297, 964; doi 10.1126/science.1075997.

6. Zhang Y., Han Y., Taylor J. A., Aizenberg J., Yang S., Polym. Mater. Sci. Eng., 2006, 95, 252.

7. Santer S., Ruhe J., Polym. J., 2004, 45, 8279; doi 10.1016/j.polymer.2004.09.085

8. Patra M., Linse P., Nano Lett., 2006, 6, 133; doi 10.1021/nl051611y.

9. Petrus P., Lísal M., Brennan J. K., Langmuir, 2010, 26, 3695; doi 10.1021/la903200j ibid. 14680; doi 10.1021/la102666g

10. Li C., Han J., Ryu C. Y., Benicewicz B. C., Macromolecules, 2006, 39, 3175; doi 10.1021/ma051983t

11. Harton S.E., Kumar S.K., J. Polym. Sci., Part B: Polym. Phys., 2008, 46, 351; doi 10.1002/polb.21346

12. Szleifer I., Yerushalmi-Rozen R., Polym. J., 2005, 46, 7803; doi 10.1016/j.polymer.2005.05.104

13. Alexander S., J. Phys., 1977, 38, 983; doi 10.1051/jphys:01977003808097700

14. de Gennes P.G., Macromolecules, 1980, 13, 1069; doi 10.1021/ma60077a009.

15. de Gennes P.G., Scaling Concepts in Polymer Physics. Cornell University Press, Ithaca, 1979.

16. Milner S.T., Science, 1991, 251, 905; doi 10.1126/science.251.4996.905.

17. Fleer G.J., Cohen Stuart A. A., Scheutjens J.M.H.M., Cosgrove T., Vincent B., Polymers at Interfaces. Chapman and Hall, London, 1993.

18. Carignano M.A., Szleifer I., J. Chem. Phys., 1995, 102, 8662; doi $10.1063 / 1.468968$.

19. Ye Y., McCoy J.D., Curro J.G.J., J. Chem. Phys., 2003, 119, 555; doi:10.1063/1.1577325.

20. Borówko M., Rżysko W., Sokołowski S., Staszewski T., J. Chem. Phys., 2007, 126, 214703; doi $10.1063 / 1.2743399$

21. Borówko M., Rżysko W., Sokołowski S., Staszewski T., J. Phys. Chem. B, 2009, 113, 4763; doi $10.1021 /$ jp811143n.

22. Milchev A., Egorov S. A., Binder K., J. Chem. Phys., 2010, 132, 184905; doi 10.1063/1.3414996.

23. Lai P.Y., Binder K., J. Chem. Phys., 1992, 97, 586; doi $10.1063 / 1.463554$.

24. Klatte S.J., Beck T.L., J. Chem. Phys., 1996, 100, 5936; doi 10.1063/1.467239

25. Grest G.S., J. Chem. Phys., 1996, 105, 5532; doi:10.1063/1.472395.

26. Kreer T., Metzger S., Müller M., Binder K., Baschnagel J., J. Chem. Phys., 2004, 120, 4012; doi $10.1063 / 1.1642615$

27. Lippa K.A., Sander L.C., Mountain R.D., Anal. Chem., 2005, 77, 7862; doi:10.1021/ac051085v

28. Pastorino C., Binder K., Keer T., Müeller M., J. Chem. Phys., 2006, 124, 064902; doi $10.1063 / 1.2162883$

29. Ohno K., Sakamoto T., Mingawa T., Okabe Y., Macromolecules, 40, 723; doi $10.1021 / \mathrm{ma} 0613234$.

30. Rafferty J.L., Siepmann J.I., Schure M.R., J. Chromatogr. A, 2009, 1216, 2320; doi $10.1016 /$ j.chroma.2008.12.088

31. MacDowell L. G., Müeller M, J. Chem. Phys., 2006, 124, 084907; doi 10.1063/1.2172597

32. Karaikos E., Bitsanis I. A., Anastasidis S. H., J. Polym. Sci.: Part B: Polym. Phys., 2009, 47, 2449. 
33. Hsu H.-P., Paul W., Binder K., J. Chem. Phys., 2010, 133, 134902; doi 10.1063/1.3495478

34. Weinhold J. D., Kumar S. K., J. Chem. Phys., 1994, 101, 4312; doi 10.1063/1.467481

35. Cao D. P., Wu J.-Z., Langmuir, 2005, 21, 9786; 2006, 22, 2712; doi $10.1021 /$ la051453q

36. Cao D. P., Wu J.-Z., J. Chem. Phys., 2004, 121, 4210; doi 10.1063/1.1774983.

37. Li A., Cao D. P., Wu J.-Z., J. Chem. Phys., 2005, 122, 174708; doi $10.1063 / 1.1886685$

38. Jiang T., Li Z., Wu J.-Z, Macromolecules, 2007, 40, 334; doi 10.1021/ma061939t

39. Pizio O., Patrykiejew A., Sokołowski S., J. Phys. Chem. C., 2007, 111, 15743; doi 10.1021/jp0736847.

40. Patrykiejew A., Sokołowski S., Tscheliessnig R., Fischer J., Pizio O., J. Phys. Chem. B, 2008, 112, 4552; doi $10.1021 /$ jp710978t

41. Pizio O., Borówko M., Rżysko W., Staszewski T., Sokołowski S., J. Chem. Phys., 2008, 128, 044702; doi: $10.1063 / 1.2829247$

42. Borówko M., Patrykiejew A., Sokołowski S., Staszewski T., Collect. Czech. Chem. Commun., 2010, 75, 221.

43. Yu Y.-X., Wu J.-Z., J. Chem. Phys, 2002, 117, 2368; doi 10.1063/1.1491240; ibid. 10156; doi $10.1063 / 1.1520530$; ibid. 2003, 118, 3835; doi $10.1063 / 1.1539840$

44. Holovko M., Vakarin E., Duda Yu., Chem. Phys. Lett., 1995, 233, 420; doi: $10.1016 / 0009-2614(94) 01480-J$

45. Pizio O., Sokołowski S., Phys. Rev. E, 1996, 53, 820; doi 10.1103/PhysRevE.53.820

46. Descas R., Sommer J.-U., Blumen A., Macromol. Theory Simul., 2008, 17, 429; doi $10.1002 /$ mats. 200800046

47. Chremos A., Self Assembly in Soft Matter, PhD Thesis, University of Edinburgh, Edinburgh, 2008, https://www.era.lib.ed.ac.uk/bitstream/1842/2686/1/Chremos\%20A\%20PhD\%20thesis\%2009.pdf

48. Coluzza I., Hansen J.-P., Phys. Rev. Lett., 2008, 100, 016104; doi 10.1103/PhysRevLett.100.016104

49. Xu X., Cao D. P., J. Chem. Phys., 2009, 130, 164901; doi:10.1063/1.3119311.

50. Weeks J. D., Chandler D., Andersen H. C., J. Chem. Phys., 1971, 54, 5237; doi $10.1063 / 1.1674820$.

51. Dimitrov D., Milchev A., Binder K., Macromol. Symp., 2007, 252, 47; doi $10.1002 /$ masy.200750605

52. Pastorino C., Binder K., Müller M., Macromolecules, 2009, 42, 401; doi 10.1021/ma8015757.

53. Martin J. I., Wang Z.-G., J. Phys. Chem., 1995, 99, 2833; doi 10.1021/j100009a045

54. Miller S. T., Witten T. A., Cates M. E., Macromolecules, 1988, 21, 2610; doi $10.1021 / \mathrm{ma00186a051}$.

55. Skvortsov A. M., Gorbunov A. A., Pavlushkov I. V., Zhoulina E. B., Borisov O. V., Priamitstn V. A., Polym. Sci. USSR, 1988, 30, 1706; doi 10.1016/0032-3950(88)90393-0.

56. Laradji M., Guo H., Zukermann M .J., Phys. Rev. E, 1994, 49, 3199; doi 10.1103/PhysRevE.49.3199

57. He G.-L., Merlitz H., Sommer J.-U., Wu C.-X., Eur. J. Phys. E, 2007, 24, 325; doi:10.1140/epje/i2007-10243-y

58. Bryk P., Sokołowski S., J. Chem. Phys., 2004, 121, 11314; doi $10.1063 / 1.1814075$

\title{
Зміни у структурі зв'язаних ланцюгових молекул згідно передбачень методу функціоналу густини
}

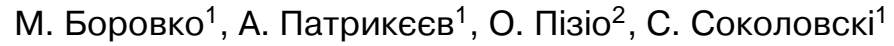 \\ 1 Відділ моделювання фізико-хімічних процесів, університет Марії Кюрі-Склодовської, \\ 20031 Люблін, Польща \\ 2 Інститут хімії УГАМ, 04360 Мехіко, Мексика
}

Ми використовуємо один із варіантів теорії функціоналу густини для вивчення змін у висоті зв'язаного шару ланцюгів, утворених з'єднаними сферичними сегментами, із змінними довжиною та поверхневою густиною ланцюгів. Для моделі, у якій взаємодії між сегментами та молекулами розчинника $є$ такими самими як між молекулами розчинника, нами виявлено два ефекти, які не спостерігалися у попередніх дослідженнях. За певних умов і при низьких поверхневих концентраціях ланцюгів висота зв'язаного шару може досягати мінімуму. Крім того, для деяких систем спостерігається що при збільшенні температури висота шару ланцюгів може зменшуватися.

Ключові слова: щітка, адсорбція, теорія функціоналу густини, масштабування 


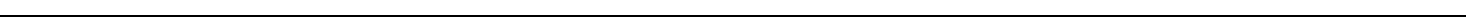

